\title{
The PROactive innovative conceptual framework on physical activity
}

\author{
Fabienne Dobbels ${ }^{1}$, Corina de Jong ${ }^{2,13}$, Ellen Drost ${ }^{3,13}$, Janneke Elberse ${ }^{4,13}$, \\ Chryssoula Feridou ${ }^{5,13}$, Laura Jacobs ${ }^{1,13}$, Roberto Rabinovich ${ }^{3}$, Anja Frei ${ }^{6}$, \\ Milo A. Puhan', Willem I. de Boer ${ }^{4}$, Thys van der Molen², Kate Williams ${ }^{7}$, \\ Hillary Pinnock ${ }^{8}$, Thierry Troosters ${ }^{9}$, Niklas Karlsson ${ }^{10}$, Karoly Kulich ${ }^{11}$, \\ Katja Rüdell ${ }^{7}$ and the PROactive consortium ${ }^{12}$
}

\begin{abstract}
Affiliations: ${ }^{1}$ Health Services and Nursing Research, University of Leuven, Leuven, Belgium. ${ }^{2}$ Dept of General Practice, University Medical Center Groningen, University of Groningen, Groningen, The Netherlands. ${ }^{3}$ ELEGI Laboratory, Center for Inflammation Research, University of Edinburgh, Edinburgh, UK. ${ }^{4}$ Lung Foundation Netherlands, Amersfoort, The Netherlands. ${ }^{5}$ Dept of Critical Care Medicine and Pulmonary Services, Thorax Foundation, Athens, Greece. ${ }^{6} \mathrm{H}$ orten Centre and Institute of Social and Preventive Medicine, University of Zurich, Zurich, Switzerland. ${ }^{7}$ Outcomes and Evidence, Global Health and Value, Pfizer, Walton Oaks, UK. ${ }^{8}$ Allergy and Respiratory Research Group, Centre for Population Health Sciences, University of Edinburgh, Edinburgh, UK. ${ }^{9}$ Dept of Rehabilitation Sciences, University of Leuven, Leuven, Belgium. ${ }^{10}$ AstraZeneca, Research and Development, Health Economics and Outcomes Research, Medical Science, Mölndal, Sweden. ${ }^{11}$ Patient Reported Outcomes Division, Novartis, Basel, Switzerland. ${ }^{12}$ For a list of the PROactive consortium members see the Acknowledgements section. ${ }^{13}$ These authors equally contributed.
\end{abstract}

Correspondence: Fabienne Dobbels, Health Services and Nursing Research, Dept of Public Health and Primary Care, University of Leuven (KU Leuven), Kapucijnenvoer 35/4, B-3000 Leuven, Belgium.

E-mail: fabienne.dobbelsamed.kuleuven.be

ABSTRACT Although physical activity is considered an important therapeutic target in chronic obstructive pulmonary disease (COPD), what "physical activity" means to COPD patients and how their perspective is best measured is poorly understood. We designed a conceptual framework, guiding the development and content validation of two patient reported outcome (PRO) instruments on physical activity (PROactive PRO instruments).

116 patients from four European countries with diverse demographics and COPD phenotypes participated in three consecutive qualitative studies (63\% male, age mean \pm SD $66 \pm 9$ years, 35\% Global Initiative for Chronic Obstructive Lung Disease stage III-IV). 23 interviews and eight focus groups $(\mathrm{n}=54)$ identified the main themes and candidate items of the framework. 39 cognitive debriefings allowed the clarity of the items and instructions to be optimised.

Three themes emerged, i.e. impact of COPD on amount of physical activity, symptoms experienced during physical activity, and adaptations made to facilitate physical activity. The themes were similar irrespective of country, demographic or disease characteristics. Iterative rounds of appraisal and refinement of candidate items resulted in 30 items with a daily recall period and 34 items with a 7-day recall period.

For the first time, our approach provides comprehensive insight on physical activity from the COPD patients' perspective. The PROactive PRO instruments' content validity represents the pivotal basis for empirically based item reduction and validation.

@ERSpublications

Conceptual framework as basis of PROactive PRO instruments to assess physical activity from COPD patient perspective http://ow.ly/ytJoS

For editorial comments see page 1110 .

Received: Jan 072014 | Accepted after revision: June 152014 | First published online: July 172014

Support statement: This study was funded by the Innovative Medicines Initiative Joint Undertaking (IMI-JU) PROactive project (GA\#115011).

Conflict of interest: Disclosures can be found alongside the online version of this article at erj.ersjournals.com

Copyright OERS 2014. ERJ Open articles are open access and distributed under the terms of the Creative Commons Attribution Non-Commercial Licence 4.0. 


\section{Introduction}

Chronic obstructive pulmonary disease (COPD) affects $\sim 10 \%$ of people and represents the third leading cause of death worldwide $[1,2]$. Besides chronic airflow limitation and a range of pathological changes in the lung, extrapulmonary consequences contribute significantly to disease progression and different disease phenotypes [3]. Reduced physical activity might trigger these extrapulmonary consequences, and is associated with an increased risk of co-morbidities, (re)hospitalisation, and all-cause or respiratory mortality [4-8].

Despite the paucity of data, it is intuitively clear that physical activity constitutes an important outcome not only to clinicians, but also to COPD patients, given the direct impact of physical activity problems on their daily functioning. For instance, the large multinational PERCEIVE (Perception of Exacerbations of Chronic Obstructive Pulmonary Disease) study conducted telephone interviews with $>1000$ patients, and found that, besides shortness of breath (78\%), COPD patients most frequently complained about their inability to complete the activities they enjoy because of their illness [9]. Improvement in physical activity might therefore be regarded as an important patient-centred therapeutic target for both pharmacological and nonpharmacological interventions.

The guidelines on patient reported outcome (PRO) instrument development put forward by the US Food and Drug Administration (FDA) clearly indicate that PRO instruments should be based upon a conceptual framework [10]. The European Medicines Agency (EMA) defines a PRO as "any outcome directly evaluated by the patient and based on the patient's perception of a disease and its treatment(s)" [11]. A conceptual framework defines the PRO concept under investigation in an unambiguous way and visualises the concept(s) measured by the PRO instrument in a diagram representing the relationships between the main concept, the domains and/or sub-domains, from which items and their scoring can be derived [10, 12]. In the absence of such a framework, it is questionable what exactly a PRO measures, which also has repercussions for labelling claims. Thus, a PRO instrument should be well-thought out and planned, rigorously developed and have good content validity, demonstrating that the PRO instrument's items and domains are appropriate and comprehensive, relative to its intended measurement concept, population and use $[10,13,14]$. The whole process should be steered by direct information from patients using appropriate qualitative research methodology.

Many self-reported instruments on physical activity or related concepts have been published to date. Recent systematic reviews, however, highlighted that their content and validity for use in COPD patients is questionable due to the lack of a conceptual framework underpinning the items [15, 16]. Clinicians and researchers often cite the physical activity definition of CASPERSEN et al. [17] as "any bodily movement produced by skeletal muscles resulting in energy expenditure beyond resting energy". While this definition has value in that it reflects a crucial aspect of physical activity in terms of maintenance of health, it is clear that this definition does not capture all aspects or "domains" of the concept that patients with COPD deem relevant. A systematic review specifically looking for conceptual frameworks on physical activity could not find any useful frameworks that could guide the development of a new PRO instrument on physical activity [18]. Therefore, the Innovative Medicines Initiative PROactive project aimed to design a conceptual framework underpinning the item generation and content validation process of the PROactive PRO instruments to capture physical activity from the perspective of patients with COPD [19].

\section{Methods}

In concordance with the International Society for Pharmacoeconomics and Outcomes Research (ISPOR) recommendations on establishing content validity of newly designed PRO instruments [13, 14], three steps were completed using a qualitative research methodology to: 1) elicit the key themes to inform the conceptual framework; 2) guide the item development process; and 3) assess the patients' understanding of the draft PROactive PRO instruments. Reporting of the methodology and results are in line with the Consolidated Criteria for Reporting Qualitative Studies guidelines [20].

\section{Design}

Step 1 used a grounded theory approach [21-23]. Semi-structured, one-to-one-interviews and focus groups with patients representing the broad spectrum of COPD were conducted to unravel their "lived experience" or detailed account of physical activity. While interviews provide in-depth information about an individual's experience, focus groups allow participants to compare their experiences and use quotes from others as cues to express their own views [20]. Examples of core questions asked include: What does physical activity mean to you? Do you experience limitations in your activities? If so, what are these? 
Step 2 combined content analysis of the quotes from patients participating in step 1 with expert input and extensive literature searches $[15,16,24,25]$ to create the items, response options and recall period for the PROactive PRO instruments.

Step 3 consists of cognitive debriefings of the initially derived items and instructions to target the mental processes respondents use when completing PRO instruments (i.e. comprehension, retrieval, judgment and response) $[26,27]$. "Thinking aloud" and probing techniques were applied, inviting a patient to verbalise any word in their minds when executing a task [28]. Examples of questions asked include: Can you explain in your own words what this question means to you? What were you thinking when reading this question? Do the response options make sense to you? Is this question relevant for you based on your own experience of physical activity?

\section{Sample and setting}

In each step, we enrolled patients from four different countries with varying geographical characteristics and care settings (i.e. the Netherlands, UK, Belgium and Greece), using the following inclusion criteria: age $\geqslant 40$ years, physician's diagnosis of COPD, fluent in the local language, and willing and able to provide written informed consent. Exclusion criteria were: age $<40$ years, institutionalised patients (nursing home or psychiatric unit), patients with documented dementia or other mental impairment, patients in palliative care, or patients with major co-morbidities that, in the physician's opinion, would make it difficult to capture aspects of physical activity that are specific for COPD (e.g. amputation and advanced heart failure).

In order to capture the heterogeneity of COPD disease and guarantee sample representativeness, a multidisciplinary expert panel was held during the 2009 European Respiratory Society Congress to define the key demographic, clinical and psychosocial phenotyping criteria and their measurement, as well as the suggested quota of patients fulfilling each criterion (table 1). The agreed measurement methodology is described in detail elsewhere [29, 30].

The main characteristics for theoretical sampling were age (at least $30 \%$ aged $<60$ years), sex (at least $40 \%$ female) and $60-70 \%$ of patients in Global Initiative for Chronic Obstructive Lung Disease (GOLD) severity classification II and III [31], as these patients are more likely to participate in future drug development studies implementing the PROactive PRO instruments. Eligible patients were identified via postings on websites of patient organisations, screening of databases of secondary or tertiary care hospitals and through a primary care network of general practitioners.

\section{Development of interview guides and training of the interviewers}

The interview guides for all three steps were developed in an iterative fashion based on qualitative research literature, expert input and pilot testing. Interviewers not involved in direct patient care were trained by two experienced qualitative researchers over four non-consecutive days using role play, hands-on practice with volunteering patients, and feedback on pilot interviews.

\section{Ethics}

The project's ethics board and Patient Input Platform [32] and the research ethics committees of the participating centres approved the protocol.

\section{Transcription and translation}

Interviews were audiotaped and transcribed verbatim by the interviewer. All elements that could lead to the participant's identification were removed (e.g. names and places). Given the project's multinational context, transcripts were translated by a certified translation company into English prior to data analysis, using established guidelines [33]. Translations were checked to ensure that no nuances or meaningful quotes were lost or altered. The conceptual framework and corresponding items were designed in English, but translated into the study languages prior to each round of cognitive debriefings. The final item lists are available in English, Dutch (for the Netherlands and Belgian) and Greek.

\section{Statistical analysis}

SAS (version 9.3; SAS Institute Inc., Cary, NC, USA) was used to summarise the sample characteristics descriptively. The computer programme Atlas.ti version 6.0 (www.atlasti.com/index.html) facilitated the qualitative data analysis.

\section{Step 1: eliciting the key themes to inform the conceptual framework}

The one-to-one transcripts were first read individually, marking text fragments referring to physical activity, and designing a draft proposal of a coding dictionary. Proposed codes were discussed with the team, after which the agreed upon coding dictionary was programmed into the Atlas.ti Software. A first round of 
TABLE 1 Phenotyping criteria, measurement and quota for the one-to-one interviews, focus groups and cognitive debriefings

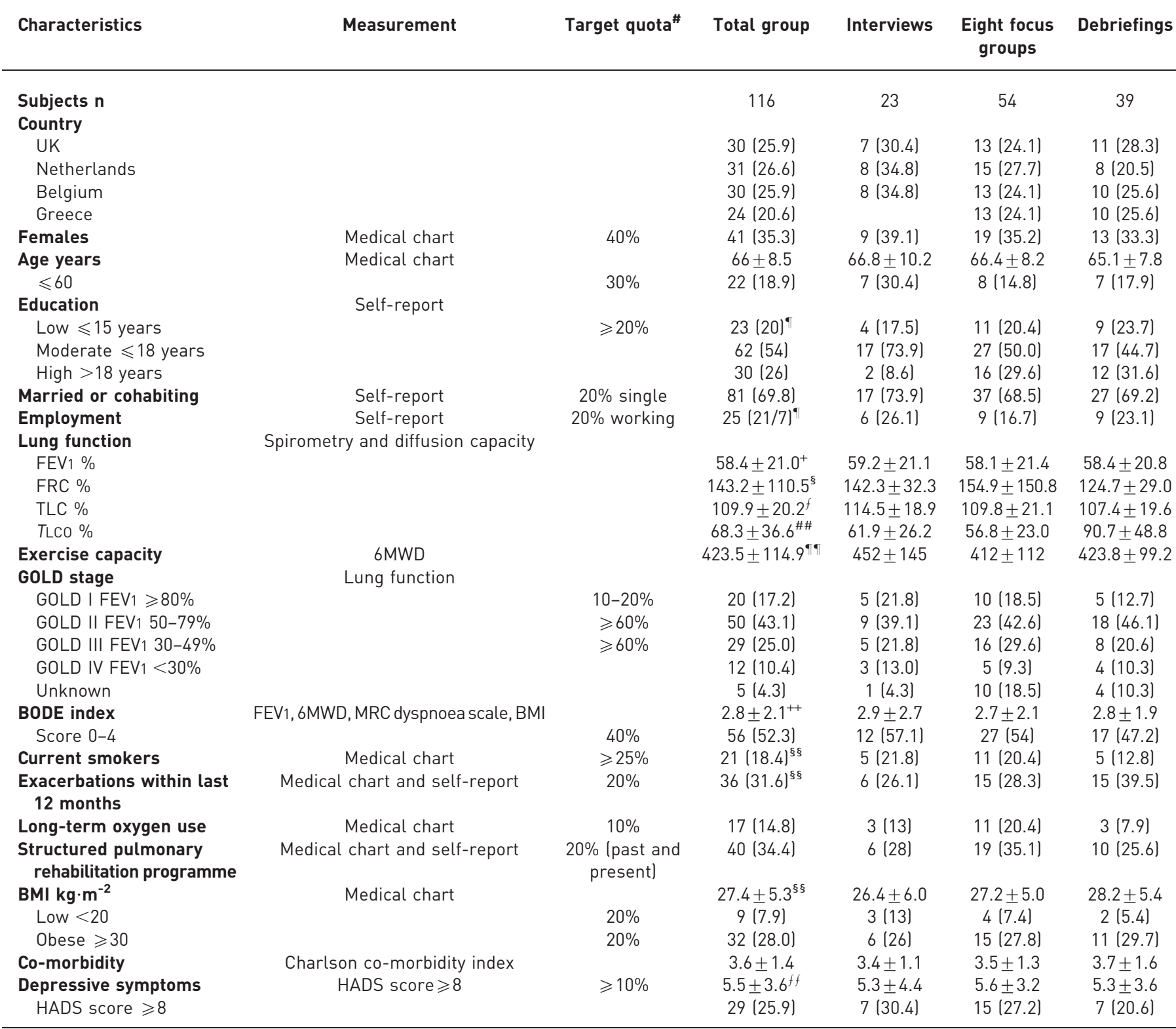

Data are presented as $\mathrm{n}(\%)$ or mean $\pm \mathrm{SD}$, unless otherwise stated. The phenotyping criteria methodology is described elsewhere [29, 30]. FEV1: forced expiratory volume in $1 \mathrm{~s}$; FRC: functional residual capacity; TLC: total lung capacity; TLCo: transfer factor of the lung for carbon monoxide; GOLD: Global Initiative for Chronic Obstructive Lung Disease; BODE: body mass index (BMI), airflow obstruction, dyspnoea, exercise capacity; HADS: Hospital Anxiety and Depression Scale; 6MWD: 6-min walk distance; MRC: Medical Research Council. " : determined based on consensus among interdisciplinary experts in chronic obstructive pulmonary disease. Targets were only used to guarantee sample representativeness by ensuring that patients with widely varying demographic, disease and psychosocial characteristics were sufficiently represented. ${ }^{\uparrow}$ : $\mathrm{n}=115$; ${ }^{+}: \mathrm{n}=113 ;{ }^{\S}: \mathrm{n}=99 ;{ }^{f}: \mathrm{n}=102 ;{ }^{\# \#}: \mathrm{n}=107 ;{ }^{\text {ศศ }}: \mathrm{n}=110 ;{ }^{++}: \mathrm{n}=107 ;{ }^{\S \S}: \mathrm{n}=114 ;{ }^{f f}: \mathrm{n}=112$.

coding of all interviews was performed independently within a 14-day period by pairs of team members, followed by a second round of coding using a slightly revised coding dictionary. A similar stepwise approach was used to analyse the focus groups. Codes for which saturation was reached were classified into meaningful themes, representing the different aspects of the concept physical activity (i.e. topics addressed by the majority of patients and supported by a large number of quotes). Regular meetings with the entire team (interviewers and non-interviewers) were scheduled to avoid interpretation bias. Results were discussed until consensus on the conceptual framework was reached. The framework was subsequently endorsed by the members of the project's consortium, the advisory board, ethics board and the Patient Input Platform [32]. 
Step 2: item development process

Guided by the themes depicted in the conceptual framework, the participants" "quotes" from the interviews and focus groups were used to form the wording of the items, following a sequence of drafting, review, modification, re-review and consensus. Although the qualitative data formed the backbone for decision making, the team also consulted evidence on possible response options, optimal recall period, and wording of items used in existing self-reported instruments $[15,16,24,25]$. Revisions made to the original set of draft items were documented in an item tracking matrix.

\section{Step 3: assess the patients' understanding of the draft PROactive PRO instruments}

Comments made during the cognitive debriefings on the clarity of wordings, instructions and response options were tabulated by each interviewer into the following categories: 1) instructions were not understood correctly; 2) respondents did not understand the item and required examples for clarification; and 3) respondents encountered difficulties with the response options. After the first round of debriefings, the English version item pool was revised, incorporating all remarks made by at least two participants. Debriefing on the updated version was undertaken by three native English speaking patients before completing the translations, followed by a new round of debriefings in each country. Remarks made during this second round of debriefings were discussed iteratively with the team members and experts, until the final item list was approved and endorsed by all consortium partners. The Flesch-Kincaid formula was used to evaluate readability of the item lists using an online calculator [34]. Reading ease is expressed by a score from $0-100$, with higher scores indicating a better readability. A score between 60 and 70 is easily understood by 13-15-year-old students, while a score of $<30$ is best understood by university graduates. Grade level is also estimated, for instance, with a grade 8 reading level corresponding with an average UK or US student of 13-14 years of age in education year 8 [35].

\section{Results}

23 one-to-one interviews, eight focus groups with 54 patients and 39 debriefings were conducted (total sample $\mathrm{n}=116,63 \%$ male, age mean \pm SD $66 \pm 9$ years, $35 \%$ GOLD stage III-IV) (table 1). Additional phenotyping characteristics are presented in table 1 .

\section{Step 1: eliciting the key themes to inform the conceptual framework}

Figure 1 represents the three core themes and their respective sub-themes directly reflecting the patients' experience of physical activity, i.e. "amount of physical activity" (what activities they do), "symptoms during physical activity" (how do they feel when doing these activities), and "need for physical adaptations" (how they perform these activities) (fig. 1). The activities affected first were climbing the stairs and walking (first "uphill" and then also on the flat). Yet, patients reported that with disease progression almost all physical activities of daily life became affected, such as carrying objects, household activities and, ultimately, self-care activities such as dressing or bathing. When being physically active, patients also noticed symptoms including breathlessness, physical exhaustion, fatigue or lack of strength. Some patients also experienced leg problems (including leg fatigue). Patients noticed variability in good and bad days, reported that symptoms are unpredictable and gradually worsen over time. Patients adapt their physical activities by pacing or slowing down, interrupting their activities to take a break to recover, or allowing a longer recuperation period. Some patients also use aids (e.g. a rollator or electrical bike) to facilitate physical activities, but a lot of activities need to be given up over time as patients are no longer able to perform them. Reduced amount of activities, activity-related symptoms and physical adaptation mechanisms are all interrelated, as illustrated by the return arrows. Table 2 shows which themes are mentioned most frequently and table 3 presents examples of quotes supporting the themes and their interrelationships.

External factors such as humid air, extreme temperatures and disease-related factors, including co-morbid conditions (e.g. back pain) and exacerbations of their COPD, may aggravate problems with physical activity. As an emotional or social consequence of their activity-related problems, some patients reported feeling sad, frustrated, dependent or isolated and family members/friends/other people did not understanding they could no longer do what they used to do, while other patients received practical help in carrying out activities. Although these influencing factors and consequences are important, they do not capture the concept of physical activity directly. Therefore, they were not considered part of the conceptual framework.

Themes were remarkably similar irrespective of country, sex, age and GOLD stage. This illustrates that physical activity is probably a universally applicable concept that is relevant for the majority of patients with COPD, and that patients use a common language to talk about physical activities (table 2). The activity "cycling" represented an exception. Patients in the Netherlands, and to a lesser extent in Belgium, noted a reduced ability to cycle, while this activity was not mentioned in the UK and Greece, rendering this activity an unsuitable candidate item. Although all patients noticed problems when having to walk uphill, cognitive 


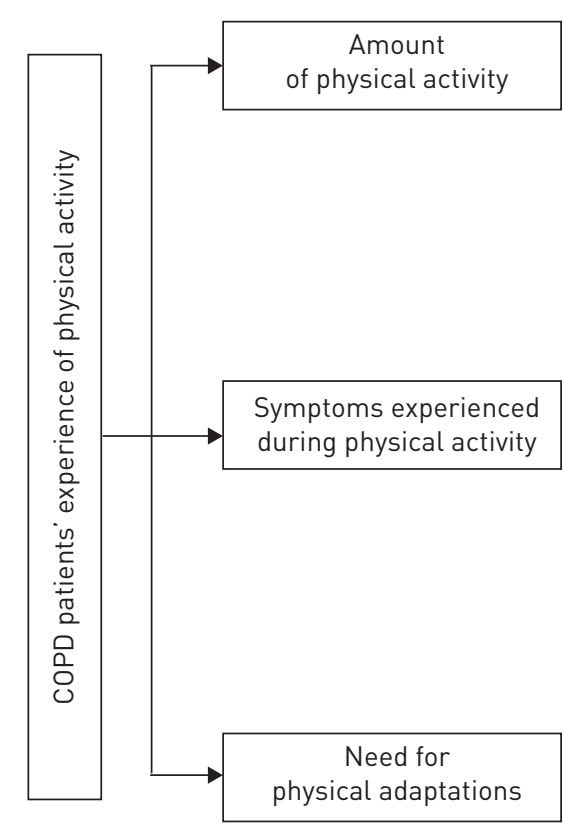

How much walking did you do outside today? How many tasks did you do inside the house today? How many tasks did you do outside the house today? How much physical leisure activity did you do today? How much difficulty did you have getting dressed today? How much difficulty did you have with carrying things you needed to today? How much difficulty did you have climbing stairs today? (If climbed stairs today) How much difficulty did you have showering/bathing today? (Depending on whether patient usually baths or showers and whether they did this]

How much difficulty did you have bending over today?

How much difficulty did you have washing today?

How much difficulty did you have walking up a small slope today?

How much difficulty did you have when hurrying today?

In the past $\mathbf{7}$ days, how much difficulty did you have getting out and about?

How breathless were you in general during your activities today?

How often did you lack physical strength to do things today?

How often did you experience lack of strength in your legs during your physical activity today?

How tired were you in general during your activities today?

How breathless were you when walking on level ground inside and outside today?

How breathless were you when climbing stairs today? (If climbed stairs today)

In the past $\mathbf{7}$ days, how often did you cough during your physical activities?

How often did you avoid doing activities because of your lung problems today? How often did you have to take breaks during your physical activities today?

How often did you have to pace yourself during your physical activities today?

How often did your lung problems prevent you from doing activities that you needed to do today? How often did you have to slow down while walking today?

How much time did you need to recover from your physical activities today?

Did you need to spread your activities throughout the day today because of your lung problems?

Did your lung problems stop you from doing the physical activities that you wanted to do today?

How many puffs of [insert name of rescue medication here] in addition to your regularly used

medication did you need to take for your physical activities today? (If on medication only)

Did you need to consider your lung problems when you planned your activities today?

How much help from others did you need with any of your activities today?

In the past $\mathbf{7}$ days, how often did you exercise to maintain or improve your physical condition?

In the past 7 days, how often did you use aids to facilitate your physical activities?

In the past 7 days, how often did you overexert yourself during your practical activities?

FIGURE 1 Conceptual framework on chronic obstructive pulmonary disease (COPD) patients' experiences of physical activity. Items for the clinic visit patient reported outcome (PRO) are similar to the items of the PRO to be completed on a daily basis, with the exception of the items in bold, which only appear in the clinic visit PRO. In addition, examples to clarify the meaning of some items are left out due to space restrictions.

debriefing revealed that the meaning of "uphill" differed between countries (e.g. a small slope versus a steep hill). Hence, this observation warranted the need for careful wording of the item, so that "uphill" has the same meaning irrespective of geographical location.

\section{Step 2: item development process}

Various versions of the item bank were drafted between August and November 2010. First, 1466 candidate items were extracted from all transcripts as well as existing instruments, and tabulated (table 2) [15, 16]. Each candidate item was carefully reviewed by the team for eligibility, resulting in a reduced list of 951 items, which was subsequently further reduced to 448 after a second screening. Reasons for removing quotes were: 1) not capturing the main themes and subthemes (e.g. items referring to determinants, consequences or past experiences, "I used to walk much faster", "I feel embarrassed when I have to stop to catch my breath"); 2) vague formulations ("I had to become a lot calmer", "it doesn't work when I want to go faster"); 3) items with a very specific content ("I cannot go to city XX or YY anymore"); and 4) quotes referring to related, but different concepts (e.g. beliefs about or perceived ability rather than actual physical activity e.g. "I think I would be able to walk $5 \mathrm{~km}$ "). Next, quotes with similar wordings were grouped within each topic. For instance, the topic "breathlessness" included quotes grouped as "breathlessness", "out of breath" or "puffing". Another example referred to reduced amount: "it is difficult to", "I have problems to", "I am not able to". Grouped items were re-reviewed iteratively and changes were documented in detailed tables, until 30 items for a daily assessment and 34 for the 7-day clinic visit assessment were ready to undergo cognitive debriefing. Final decisions on wording were made based on the number of quotes with similar phrasings, easiness to read the items, and extensive discussions with the consortium and advisory board. 


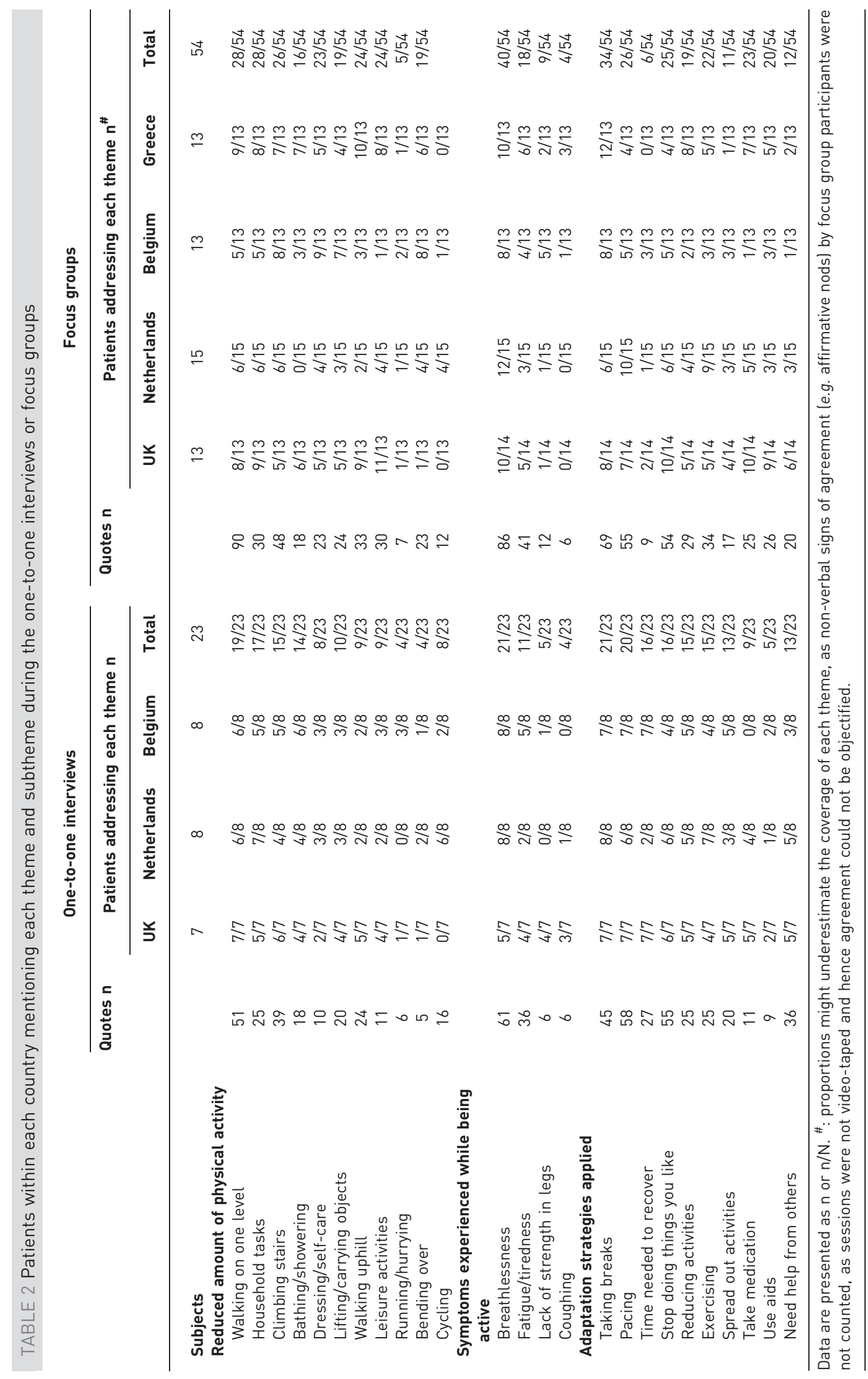




\section{TABLE 3 Examples of quotes to support the themes and subthemes of the conceptual framework}

Quotes

"But, you know, I take things easy, right. If I have to climb stairs I have more difficulty than I have here (i.e. in the hospital), you see. Today I can take the sun pretty well, I mean, I can walk, but once it goes uphill I'm in trouble. When I get home I have to climb a hill, 5 metres up. First it goes slightly up and then I have to climb stairs but then I have to go and sit down for a bit. I do feel it then. I mean, that I'm out of breath. It only takes 5 minutes, but then I have to lie down again - that's at 7 o'clock in the morning - and then I recover."

\section{(Belgium, interview 3: male, age 65 years, GOLD stage II)}

“To make myself clear, because we shouldn't lie now, I'm not at the level I want to be. To make it... you therefore divide it into breaks, you stop at the stairs with the bags and you begin again... At the stairs or, I don't know, let's say, in the street where carry the trolley from the farmers' market, let's say, it's a distance of 200 to 300 meters, 400 to 500 . Because even at the farmer' market you come and go, you come and go. You don't stay in one place, because in that case there's no point going to the farmers' market... And when I get tired, I look for a crate, let's say, at the farmers' market. If I'm tired, I take a break. I sit on the crate... oof... I say something silly to an Olympiakos fan, to a Panathinaikos fan and I start again and make it to the house. That is, I try to gain, let's say, metres, to give myself some time and to catch my breath and to raise my morale."

\section{(Greece, focus group 1: male, aged 57 years, GOLD stage II]}

"I live on my own, so my housekeeping must also be done. For example, when I have to mow the lawn, I just have a small garden and I have to mow that, and the neighbours have to too. Otherwise I get discredited so that does have to happen. And then I do that in three goes. So do a bit, and then I go back inside, lie on the bench and then, eh, then I think, yes I am back, and then I go back. Then I do it... What used to take a quarter of an hour, that garden, I now do in more than an hour and a half. I pant, then pant, so I then quit. Now I almost never want to walk. And cycling, that goes very well, but that is with a cycle with support. That is, that is the invention of the century."

\section{(Netherlands, focus group 2: male, aged 71 years, GOLD stage I)}

"Getting dressed and undressed when I'm so short of breath, I need my wife for that as well, you know. Also when you go for a bath and you're sitting there in a small room with all the steam and less oxygen... that is actually the thing l'm most sensitive to...getting my breath. So that's not something that I go and do every day, you see! I look at that as split tasks, you know. Having a bath, drying yourself and whatever, you have to divide that up as well, right. And that's bending and straightening up, isn't it. Because how else are you going to get your feet dry? And the days that I do those things I know that I mustn't plan too many other things.

(Belgium, interview 5: male, aged 64 years, GOLD stage IV)

[About social activities] "I had to give them all up. I've done a lot of work outside and that. I had the pigeons but had to gee [give] them up right away. There are a lot of things I've had to give up, you know. Well playing football, like football and that. Can't walk along the corridor, never mind run. And as I said, when you go out with your wife and she's carrying two big bags and your walking along there with nothing, people must say to themselves "oh look at that man his wife carrying the bags and he's got nothing", but at the end of the day they dinnae ken [don't know] what's happening inside you!"

(UK, focus group 2: male, aged 65 years, GOLD stage II)

"There a great deal of things that are the same. I do things and of course I am not 20 any longer, but I do things. It's normally more slowly and indeed, and then I do take a puff, but it sometimes it does take you by surprise. Er, it's happened to me a few times as if everything pinches tightly. It has happened a few times now, so I normally try to make sure that it doesn't happen again. But with extra efforts, walking on the stairs and like that, it does happen to me. Really serious things, then I think, "rest for a bit" because otherwise it won't be good. And one time it goes fine and the other time, then is [pretends to be out of breath]. That is mad, it is never actually the same."

(Netherlands, focus group 2: male, aged 63 years, GOLD stage II)

“I couldn't walk. That means that l've got a fold up wheelchair, ehm which I use quite a lot. I've now bought myself a scooter, like yours P5, which I find I am more independent on a scooter than in a wheelchair, you know, I can stop at a shop and say I'm here [laughs] and I don't have to shout "stop". So, that's the only way I can go shopping now, I can't I can't walk through a shopping centre without... the breathing is bad but it it's also it's is agony for me because your ... your struggling to breathe you have to keep stopping and people stop and look at you and you feel like dirt on the floor [laughs]. It is bad, very very bad when you can't breathe.

\section{(UK focus group 2: female, aged 70 years, GOLD stage III)}

"So, you are breathing with difficulty and you are fighting to recover from your legs and from your breathing so that you can walk another ten metres. And the same again at the next ten metres. A stop. You can't go any longer. Concerning stairs... um... um... in the morning I think and say, "I will go, let's say, two to ten steps to go there, but how will I go?" Because I must also make such stops, and I must know where to sit."

(Greece, focus group 1: male, aged 72 years, GOLD stage II)
Themes

Doing things slower

Difficulty climbing stairs

Difficulty going uphill

Taking breaks/time needed to recover

Taking breaks

Problems with walking

Difficulty climbing stairs

Tiredness

Time needed to recover

Breathlessness

Problems with tasks outside and walking Taking breaks/interrupting activities

Time needed to recover

Breathlessness

Using aids (e.g. electric bike)

Spreading and planning activities Difficulties bathing and getting dressed Breathlessness

Difficulties bending over

Needing help from others

Giving up the things you like

Problems with walking

Needing help from others

Doing things slower

Taking medication

Breathlessness

Difficulty climbing stairs

Difficulties walking

Using aids

Breathlessness

Taking breaks/interrupting activities

Leg problems

Breathlessness

Problems with walking

Taking breaks and planning activities

Time needed to recover

GOLD: Global Initiative for Chronic Obstructive Lung Disease.

In view of the recall period, two different versions of the PRO instrument were designed to measure experience with PA, i.e. a daily patient diary and a 7-day recall clinic visit version. Patients who were interviewed noted large day-to-day fluctuations in the amount of activities they could do and the level of symptoms experienced while being physically active. This necessitates a variety of adaptation mechanisms in order to overcome their limitations. For instance, patients mentioned feeling extremely breathless one day while walking, while on other days they could walk longer without feeling too breathless or without needing to take many breaks. Also, when soliciting patients' opinions on recall periods, many suggested developing an instrument that needed to be completed daily at the end of each day. These patient-generated data justify the development of a PRO instrument on COPD patients' experiences of physical activity with a 1-day recall period.

Yet, not all activities that are important to patients are conducted on a daily basis, such as household tasks or leisure activities, with some activities tending to follow a weekly cycle (e.g. cleaning the house on Friday, 
visiting friends on Tuesday, etc.). Moreover, some adaptations also refer to longer time periods, like time needed to recover, or formally prescribed exercises. In addition, many patients pointed towards fluctuations over longer periods of time on top of day to day variations, and differentiated between better and worse periods of time. For instance, some patients talked about their physical activity in the weeks before hospitalisation for an exacerbation (e.g. they could do less activities and experienced significant breathlessness and exhaustion while being active), the recovery period (slowly performing some activities again), or the impact of rehabilitation on their physical activity levels (e.g. learning new ways to adapt in order to maximise their level of physical activity without increasing their symptoms). This patient-driven information justifies the development of a PRO that is able to capture these variations over longer periods of time. Admittedly, patients had varying opinions on the best recall period. This is in line with published evidence, underscoring that there is not a "one size fits all approach" [24], and that the appropriate length of the recall period very much depends on the concept being studied. For fluctuating phenomena, such as symptoms or activities of daily living, it is recommended not to use recall periods that go beyond 7 days, justifying our choice for a 7-day recall period for the clinic visit PRO instrument [24].

Different response options were explored based on expert opinion and a critical analysis of existing literature. In line with evidence-based recommendations [25], the team decided to use a five-point labelled Likert scale, reflecting a gradually reduced amount of physical activity, increasing symptoms experienced during physical activity, and a higher need for adaptations during physical activity.

Step 3: assess the patients" understanding of the draft PROactive PRO instrument

After translating the item lists into the study languages, a first round of cognitive debriefings $(n=14)$ was conducted in December 2010 and January 2011.

Patients suggested further clarifying the concept of physical activity in the introduction. In addition, some terms were difficult to understand and needed rephrasing into more user friendly terms. Also, some response options were insufficiently discriminating (e.g. referred to almost the same degree of intensity) and revisions of response options were suggested. Some items also needed a "not applicable" option (e.g. if patients don't have stairs, they should be able to leave questions referring to the climbing stairs blank). Analysis and modification continued until consensus within the team was reached.

The main comments during the second round of debriefings $(n=22)$ referred to the response options: some items provoked a yes/no response and needed to be rephrased in order to be suitable for a five-point response option. Additional changes to the response options for the item on walking were suggested. The daily and 7-day recall period was deemed appropriate by the majority of patients. Patients' suggestions were integrated and discussed until consensus was reached on the final item list for both versions of the PRO (fig. 1). Flesch Reading Ease was 81 for the daily and 86 for the clinic visit items, corresponding with to reading level of an 8-9-year-old child, indicating that the items are easy to understand by patients with very low education and/or literacy.

\section{Discussion}

Based on direct patient input, we designed an innovative conceptual framework to understand experience with physical activity from the patients' experience. This provided the necessary conceptual basis to generate items for two PRO tools to measure physical activity in patients with COPD. Although the ISPOR guidelines on establishing content validity were not available at the start of our qualitative studies, we succeeded to rigorously adhere to all methodological requirements needed to demonstrate content validity of newly designed PRO instruments [13,14]. Joint efforts between academia, the European Federation of Pharmaceutical Industries and Associations partners, patient and professional organisations, and a smallmedium enterprise made this labour-intensive work possible.

Until now, few qualitative studies on patients with COPD have been conducted and focus almost exclusively on living with a chronic illness or symptom experience $[36,37]$. Yet, looking closer at the themes addressed it becomes obvious that patients also complained about reduced ability to execute their daily activities, symptom burden while being active and the social and emotional consequences of reduced physical activity. In contrast to our qualitative studies, none of these studies specifically focused their discussion guide on physical activity. This might, in part, explain why adaptations emerged as a prominent theme during our qualitative studies, alongside the level of physical activity and symptoms experienced while being active.

Interestingly, patients had similar experiences irrespective of demographic characteristics, severity of illness or country of origin, suggesting that physical activity is most likely a universal concept that is relevant to the majority of patients with COPD. Admittedly, although the large sample size, the extensive phenotyping and the multinational nature of our qualitative studies are major strengths of our work, we don't know if patients from other countries or continents have similar experiences. However, unpublished data of focus 
groups with US patients on a related concept (i.e. limitations in physical activity) yielded similar themes, strengthening our conviction on the concept's generalisability. Moreover, international members from the project's Patient Input Platform, as well as clinical experts participating in the advisory board, all endorsed the relevance of these overarching themes, as well as the items. Readability of the item lists, which is usually not evaluated in other self-reported instruments, is excellent, guaranteeing that patients with very low literacy will also be able to complete the PROactive PRO instruments. Future steps include quantitative work to confirm the conceptual framework, establish a conceptual model, select the most appropriate items and demonstrate further validation. This will be done in compliance with existing guidelines for PRO instrument development. As the item lists are currently only available in English for UK, Dutch for Belgium and the Netherlands and Greek for Greece, culturally sensitive translations for $>50$ languages are planned once the item selection process is completed. The PROactive consortium will also engage in discussions with regulatory authorities (FDA and EMA) to ensure the PROactive tools fulfil all criteria for inclusion in future claims of effectiveness of pharmacological and non-pharmacological interventions.

In conclusion, our qualitative studies help to understand the experience of physical activity from the patients' perspective. The design of the conceptual framework and the methodological rigor followed to generate items capturing relevant themes and document content validity will most likely spin off in PRO tools that, for the first time, are able to adequately capture this important concept.

\section{Acknowledgements}

We would like to acknowledge the members of the PROactive consortium for the outstanding contribution to this work. The PROactive consortium collaborators are as follows. Caterina Brindicci and Tim Higenbottam (Chiesi Farmaceutici S.A, Parma, Italy); Thierry Troosters, Fabienne Dobbels and Marc Decramer (Katholieke Universiteit, Leuven, Belgium); Margaret Tabberer (GlaxoSmithKline, Brentford, UK); Roberto A Rabinovich and William MacNee (University of Edinburgh, Edinburgh, UK); Ioannis Vogiatzis (Thorax Research Foundation, Athens, Greece); Michael Polkey and Nick Hopkinson (Royal Brompton and Harefield NHS Foundation Trust, London, UK); Judith Garcia-Aymerich (Municipal Institute of Medical Research, Barcelona, Spain); Milo Puhan and Anja Frei (Universität Zürich, Zurich, Switzerland); Thys van der Molen and Corina de Jong (University Medical Center, Groningen, the Netherlands); Pim de Boer (Lung Foundation Netherlands, Amersfoort, the Netherlands); Ian Jarrod (British Lung Foundation, London, UK); Paul McBride (Choice Healthcare Solution, London, UK); Nadia Kamel (European Respiratory Society, Brussels, Belgium); Katja Rudell and Frederick J. Wilson (Pfizer, Walton Oaks, UK); Nathalie Ivanoff (Almirall, Barcelona, Spain); Karoly Kulich and Alistair Glendenning (Novartis, Basel, Switzerland); Niklas X. Karlsson and Solange Corriol-Rohou (AstraZeneca AB, Mölndal, Sweden); Enkeleida Nikai (UCB, Brussels, Belgium); and Damijan Erzen (Boehringer Ingelheim, Ingelheim am Rhein, Germany).

\section{References}

1 Lopez AD, Shibuya K, Rao C, et al. Chronic obstructive pulmonary disease: current burden and future projections. Eur Respir J 2006; 27: 397-412.

2 Buist AS, McBurnie MA, Vollmer WM, et al. International variation in the prevalence of COPD (the BOLD study): a population-based prevalence study. Lancet 2007; 370: 741-750.

3 Rabe KF, Hurd S, Anzueto A, et al. Global strategy for the diagnosis, management, and prevention of chronic obstructive pulmonary disease. Am J Respir Crit Care Med 2007; 176: 532-555.

4 Garcia-Aymerich J, Lange P, Benet M, et al. Regular physical activity reduces hospital admission and mortality in chronic obstructive pulmonary disease: a population based cohort study. Thorax 2006; 61: 772-778.

Pitta F, Troosters T, Probst VS, et al. Physical activity and hospitalization for exacerbation of COPD. Chest 2006; 129: 536-544.

6 Watz H, Waschki B, Boehme C, et al. Extrapulmonary effects of chronic obstructive pulmonary disease on physical activity. Am J Respir Crit Care Med 2008; 177: 743-751.

7 Decramer M, Rennard S, Troosters T, et al. COPD as a lung disease with systemic consequences - clinical impact, mechanisms, and potential for early intervention. COPD 2008; 5: 235-256.

8 Waschki B, Kirsten A, Holz O, et al. Physical activity is the strongest predictor of all-cause mortality in patients with COPD: a prospective cohort study. Chest 2011; 140: 331-342.

9 Miravitlles M, Anzueto A, Legnani D, et al. Patient's perception of exacerbations of COPD - the PERCEIVE study. Respir Med 2007; 101: 453-460.

10 US Department of Health and Human Services, Food and Drug Administration. Guidance for industry: patientreported outcome measures: use in medical product development to support labeling claims. www.fda.gov/downloads/ Drugs/GuidanceComplianceRegulatoryInformation/Guidances/UCM193282.pdf Date last updated: December 2009. Date last accessed: April 22, 2014.

11 European Medicines Agency. Committee for medicinal products for human use. Reflection paper on the regulatory guidance for the use of health-related quality of life (HRQL) measures in the evaluation of medicinal products. www. ema.europa.eu/docs/en_GB/document_library/Scientific_guideline/2009/09/WC500003637.pdf Date last updated: July 27, 2005. Date last accessed: April 22, 2014.

12 Rothman ML, Beltran P, Cappelleri JC, et al. Patient-reported outcomes: conceptual issues. Value Health 2007; 10: Suppl. 2, S66-S75.

13 Patrick DL, Burke LB, Gwaltney CJ, et al. Content validity - establishing and reporting the evidence in newly developed patient-reported outcomes (PRO) instruments for medical product evaluation: ISPOR PRO good research practices task force report: part 1 - eliciting concepts for a new PRO instrument. Value Health 2011; 14: 967-977. 
14 Patrick DL, Burke LB, Gwaltney CJ, et al. Content validity - establishing and reporting the evidence in newly developed patient-reported outcomes (PRO) instruments for medical product evaluation: ISPOR PRO good research practices task force report: part 2 - assessing respondent understanding. Value Health 2011; 14: 978-988.

15 Frei A, Williams K, Vetsch A, et al. A comprehensive systematic review of the development process of 104 patientreported outcomes (PROs) for physical activity in chronically ill and elderly people. Health Qual Life Outcomes 2011; 9: 116

16 Williams K, Frei A, Vetsch A, et al. Patient-reported physical activity questionnaires: a systematic review of content and format. Health Qual Life Outcomes 2012; 10: 28.

17 Caspersen CJ, Powell KE, Christenson GM. Physical activity, exercise, and physical fitness: definitions and distinctions for health-related research. Public Health Rep 1985; 100: 126-131.

18 Gimeno-Santos E, Frei A, Dobbels F, et al. Validity of instruments to measure physical activity may be questionable due to a lack of conceptual frameworks: a systematic review. Health Qual Life Outcomes 2011; 9: 86.

19 PROactive COPD. www.proactiveCOPD.com

20 Tong A, Sainsbury P, Craig J. Consolidated criteria for reporting qualitative research (COREQ): a 32-item checklist for interviews and focus groups. Int J Qual Health Care 2007; 19: 349-357.

21 Creswell JW, ed. Qualitative inquiry and research design: choosing among five traditions. Thousand Oaks, Sage Publications, 1997.

22 Corbin JM, Strauss A, eds. Basics of Qualitative Research 3e: Techniques and Procedures for Developing Grounded Theory. Los Angeles, Sage Publications, 2008.

23 Starks H, Trinidad SB. Choose your method: a comparison of phenomenology, discourse analysis, and grounded theory. Qual Health Res 2007; 17: 1372-1380.

24 Stull DE, Leidy NK, Parasuraman B, et al. Optimal recall periods for patient-reported outcomes: challenges and potential solutions. Curr Med Res Opin 2009; 25: 929-942.

25 Khadka J, Gothwal VK, McAlinden C, et al. The importance of rating scales in measuring patient-reported outcomes. Health Qual Life Outcomes 2012; 10: 80

26 Collins D. Pretesting survey instruments: an overview of cognitive methods. Qual Life Res 2003; 12: 229-238.

27 McColl E, Meadows K, Barofsky I. Cognitive aspects of survey methodology and quality of life assessment. Qual Life Res 2003; 12: 217-218.

28 Charters E. The use of think-aloud methods in qualitative research an introduction to think-aloud methods. Brock Education J 2003; 12: 68-82.

29 Rabinovich RA, Louvaris Z, Raste Y, et al. Validity of physical activity monitors during daily life in patients with COPD. Eur Respir J 2013; 42: 1205-1215.

30 Van Remoortel H, Raste Y, Louvaris Z, et al. Validity of six activity monitors in chronic obstructive pulmonary disease: a comparison with indirect calorimetry. PLoS One 2012; 7: 20.

31 Global Initiative for Chronic Obstructive Lung Disease. Global strategy for the diagnosis and, management and prevention of chronic obstructive lung disease. www.goldcopd.org/uploads/users/files/GOLD_Report_2014_Jun11.pdf Date last updated: June 26, 2014. Date last accessed: April 22, 2014.

32 PROactive COPD. What is PROactive? www.proactivecopd.com/about/advisory-boards Date last updated: June 26, 2014. Date last accessed: April 22, 2014.

33 Wild D, Grove A, Martin M, et al. Principles of good practice for the translation and cultural adaptation process for patient-reported outcomes (PRO) measures: report of the ISPOR Task Force for Translation and Cultural Adaptation. Value Health 2005; 8: 94-104.

34 Readability formulas. Free Text Readability Consensus Calculator. www.readabilityformulas.com/free-readabilityformula-tests.php Date last accessed: April 22, 2014.

35 Wang LW, Miller MJ, Schmitt MR, et al. Assessing readability formula differences with written health information materials: application, results, and recommendations. Res Social Adm Pharm 2013; 9: 503-516.

36 Giacomini M, DeJean D, Simeonov D, et al. Experiences of living and dying with COPD: a systematic review and synthesis of the qualitative empirical literature. Ont Health Technol Assess Ser 2012; 12: 1-47.

37 Gysels M, Bausewein C, Higginson IJ. Experiences of breathlessness: a systematic review of the qualitative literature. Palliat Support Care 2007; 5: 281-302. 